\title{
Erratum to: Finding new physics without learning about it: anomaly detection as a tool for searches at colliders
}

\author{
M. Crispim Romão ${ }^{1} \mathbb{C}$, N. F. Castro $^{1,2, \mathrm{a}}{ }_{\mathbb{C}}$, R. Pedro $^{1}{ }^{(\mathbb{C}}$ \\ ${ }^{1}$ LIP, Av. Professor Gama Pinto 2, 1649-003 Lisbon, Portugal \\ ${ }^{2}$ Departamento de Física, Escola de Ciências, Universidade do Minho, 4710-057 Braga, Portugal
}

Received: 25 October 2021 / Accepted: 5 November 2021 / Published online: 22 November 2021

(C) The Author(s) 2021

\section{Erratum to: Eur. Phys. J. C (2021) 81:27 https://doi.org/10.1140/epjc/s10052-020-08807-w}

On further processing the simulated data used in the article for open access release, we detected a defective feature in the benchmark signals samples.

The issue affects the results of assessing the performance of the Anomaly Detection (AD) methods with the selected Beyond the Standard Model signals reported in Sect. 5Comparison of the $\mathrm{AD}$ methods for benchmark signals. The correct results are presented in Figs. 1, 2 and Table 1.
Although the performance of the AD methods changes quantitatively, their relative sensitivity to the benchmark signals is generally maintained: deep learning outperforms shallow learning and the Deep SVDD model presents the best response to the variety of new signals tested, indicating to better suit to the generic detection purpose.

The data samples were made available for open access through the Zenodo online repository for research data, with record number 5126747 [2]. Finally, the corrected version of the article is also available in arXiv [3].

The original article can be found online at https://doi.org/10.1140/ epjc/s10052-020-08807-w.

a e-mail: Nuno.Castro@ cern.ch (corresponding author) 

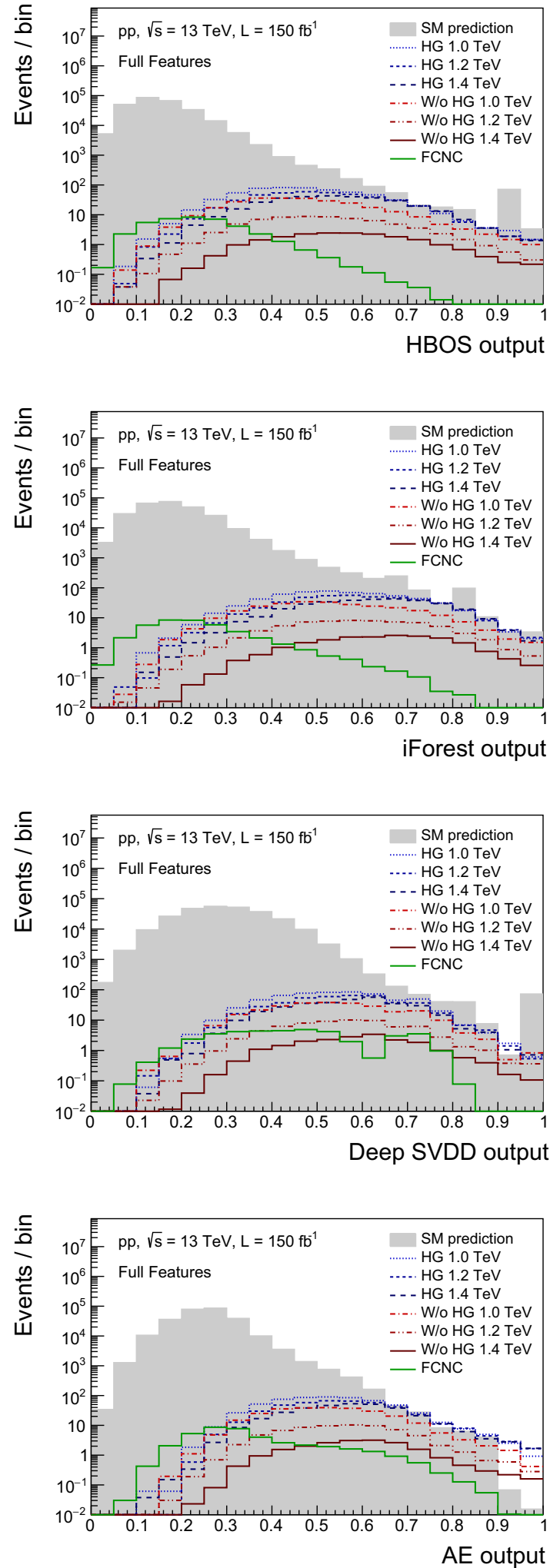

Fig. 1 Distribution of the AD discriminant for the SM prediction and each signal type: $t Z$ production by FCNC, $T \bar{T}$ production via heavy gluon or without heavy gluon for $m_{T}=\{1.0,1.2,1.4\} \mathrm{TeV}$. The dis-
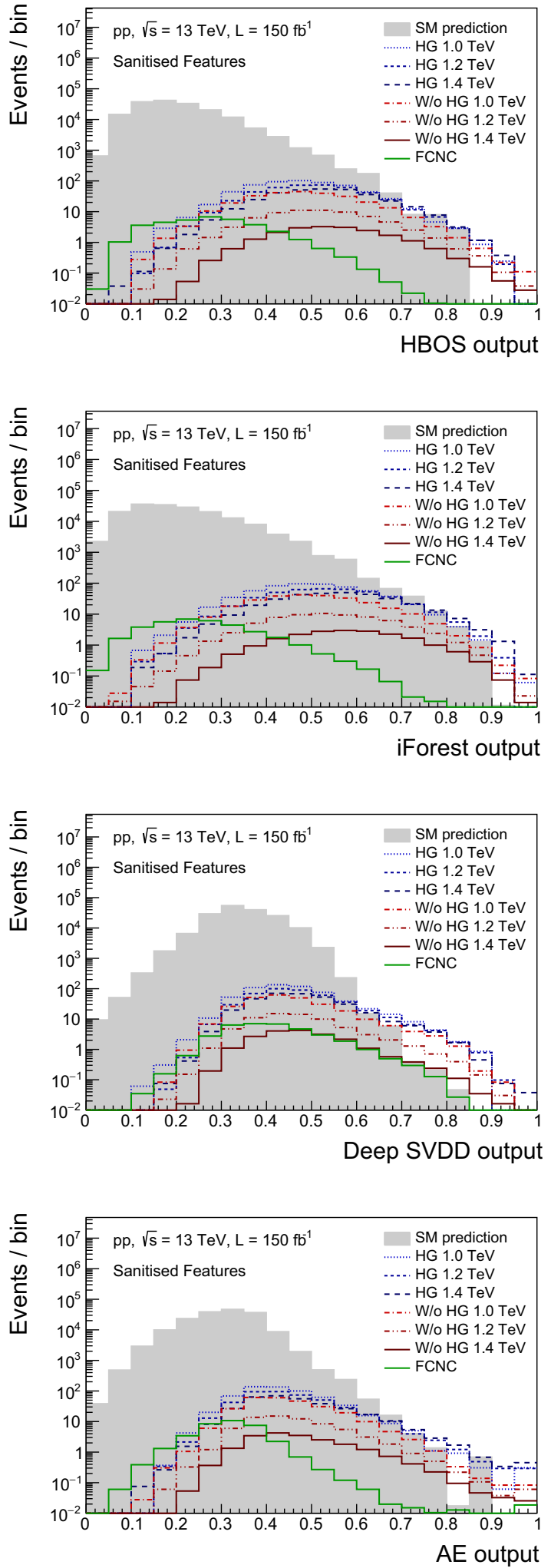

tributions are normalised to the generation cross-section and to an integrated luminosity of $150 \mathrm{fb}^{-1}$. Left: Using all features set. Right: Using sanitised features set 
Table $195 \%$ CL upper limit on the signal strength $\mu$ of each benchmark signal for the different $\mathrm{AD}$ methods using the full feature set and the sanitised set and for a dedicated supervised DNN model trained on the full feature set. The statistical uncertainties, including the effect from limited statistics in the simulated datasets, are also shown
Fig. 2 95\% CL upper limits on $\mu$ normalised to the limit obtained for the supervised DNN model

\begin{tabular}{|c|c|c|c|c|c|c|c|}
\hline \multirow[t]{3}{*}{ Model } & \multicolumn{7}{|c|}{ Benchmark signal } \\
\hline & \multirow[t]{2}{*}{ FCNC } & \multicolumn{3}{|l|}{$\mathrm{HG}$} & \multicolumn{3}{|l|}{ No HG } \\
\hline & & $1.0 \mathrm{TeV}$ & $1.2 \mathrm{TeV}$ & $1.4 \mathrm{TeV}$ & $1.0 \mathrm{TeV}$ & $1.2 \mathrm{TeV}$ & $1.4 \mathrm{TeV}$ \\
\hline \multicolumn{8}{|l|}{ Full features } \\
\hline Supervised DNN & $2.9_{-0.9}^{+1.4}$ & $0.09_{-0.03}^{+0.04}$ & $0.3_{-0.1}^{+0.2}$ & $0.17_{-0.06}^{+0.07}$ & $0.26_{-0.08}^{+0.13}$ & $1.9_{-0.8}^{+1.3}$ & $2.3_{-0.7}^{+1.1}$ \\
\hline$H_{T}$ & $60_{-20}^{+20}$ & $0.27_{-0.09}^{+0.14}$ & $0.3_{-0.1}^{+0.2}$ & $0.29_{-0.09}^{+0.16}$ & $0.8_{-0.2}^{+0.5}$ & $1.9_{-0.7}^{+0.9}$ & $3.2_{-1.0}^{+1.7}$ \\
\hline Deep SVDD & $6_{-1}^{+3}$ & $0.4_{-0.1}^{+0.1}$ & $0.4_{-0.1}^{+0.2}$ & $0.5_{-0.1}^{+0.2}$ & $0.9_{-0.3}^{+0.4}$ & $2.6_{-0.8}^{+1.2}$ & $7_{-2}^{+4}$ \\
\hline $\mathrm{AE}$ & $20_{-9}^{+4}$ & $0.25_{-0.08}^{+0.13}$ & $0.26_{-0.08}^{+0.13}$ & $0.28_{-0.09}^{+0.13}$ & $0.6_{-0.2}^{+0.2}$ & $1.4_{-0.4}^{+0.7}$ & $4_{-1}^{+1}$ \\
\hline HBOS & $60_{-20}^{+20}$ & $0.3_{-0.1}^{+0.2}$ & $0.4_{-0.1}^{+0.2}$ & $0.4_{-0.1}^{+0.2}$ & $0.8_{-0.2}^{+0.4}$ & $2.2_{-0.7}^{+1.0}$ & $5_{-1}^{+3}$ \\
\hline iForest & $70_{-20}^{+30}$ & $0.4_{-0.2}^{+0.1}$ & $0.4_{-0.1}^{+0.2}$ & $0.5_{-0.2}^{+0.2}$ & $0.9_{-0.3}^{+0.4}$ & $2.3_{-0.7}^{+1.3}$ & $6_{-2}^{+4}$ \\
\hline \multicolumn{8}{|l|}{ Sanitised features } \\
\hline Supervised DNN & $2.8_{-0.9}^{+1.3}$ & $0.22_{-0.1}^{+0.18}$ & $0.3_{-0.1}^{+0.2}$ & $0.4_{-0.2}^{+0.2}$ & $0.5_{-0.2}^{+0.5}$ & $1.8_{-0.8}^{+1.4}$ & $5_{-2}^{+5}$ \\
\hline$H_{T}$ & $50_{-10}^{+20}$ & $0.27_{-0.09}^{+0.14}$ & $0.3_{-0.1}^{+0.16}$ & $0.29_{-0.09}^{+0.16}$ & $0.8_{-0.2}^{+0.5}$ & $1.8_{-0.5}^{+1.0}$ & $3_{-1}^{+2}$ \\
\hline Deep SVDD & $6_{-2}^{+3}$ & $0.19_{-0.05}^{+0.08}$ & $0.21_{-0.05}^{+0.1}$ & $0.24_{-0.06}^{+0.11}$ & $0.36_{-0.09}^{+0.16}$ & $1.1_{-0.3}^{+0.5}$ & $3.6_{-0.9}^{+1.5}$ \\
\hline $\mathrm{AE}$ & $60_{-20}^{+30}$ & $0.9_{-0.3}^{+0.5}$ & $0.8_{-0.3}^{+0.4}$ & $0.6_{-0.2}^{+0.4}$ & $1.6_{-0.5}^{+1.0}$ & $4_{-1}^{+2}$ & $9_{-3}^{+5}$ \\
\hline HBOS & $60_{-20}^{+20}$ & $0.5_{-0.2}^{+0.3}$ & $0.5_{-0.2}^{+0.3}$ & $0.5_{-0.2}^{+0.3}$ & $1.0_{-0.4}^{+0.5}$ & $2.4_{-0.8}^{+1.5}$ & $6_{-2}^{+3}$ \\
\hline iForest & $70_{-20}^{+30}$ & $0.5_{-0.2}^{+0.2}$ & $0.5_{-0.2}^{+0.2}$ & $0.4_{-0.1}^{+0.2}$ & $1.1_{-0.4}^{+0.5}$ & $2.4_{-0.8}^{+1.2}$ & $5_{-2}^{+3}$ \\
\hline
\end{tabular}

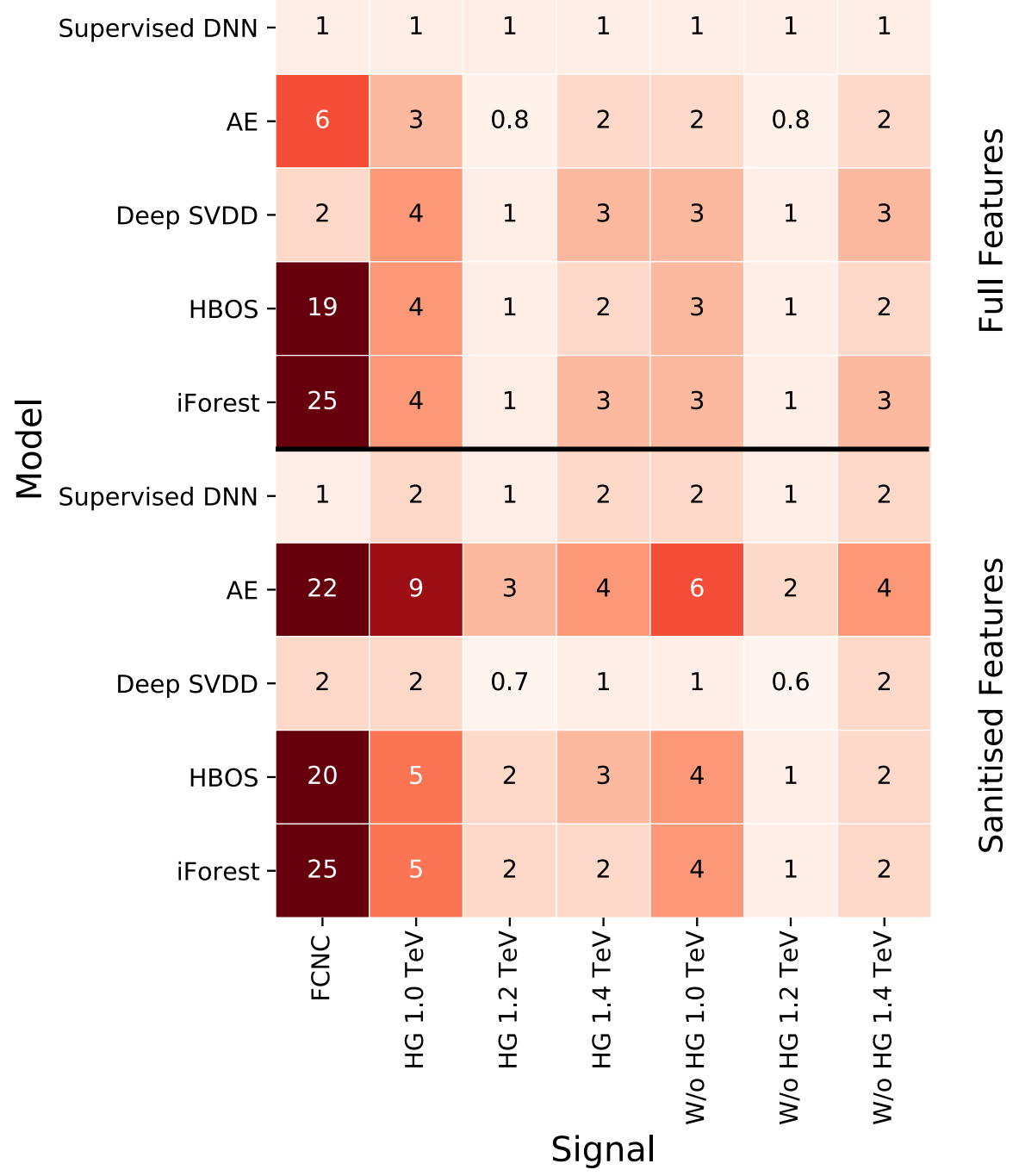


Open Access This article is licensed under a Creative Commons Attribution 4.0 International License, which permits use, sharing, adaptation, distribution and reproduction in any medium or format, as long as you give appropriate credit to the original author(s) and the source, provide a link to the Creative Commons licence, and indicate if changes were made. The images or other third party material in this article are included in the article's Creative Commons licence, unless indicated otherwise in a credit line to the material. If material is not included in the article's Creative Commons licence and your intended use is not permitted by statutory regulation or exceeds the permitted use, you will need to obtain permission directly from the copyright holder. To view a copy of this licence, visit http://creativecomm ons.org/licenses/by/4.0/.

Funded by SCOAP ${ }^{3}$.

\section{References}

1. M. Crispim Romão, N.F. Castro, R. Pedro, Finding new physics without learning about it: anomaly detection as a tool for searches at colliders. Eur. Phys. J. C 81(1), 27 (2021)

2. M. Crispim Romao, N.F. Castro, R. Pedro, Simulated pp collisions at 13 tev with 2 leptons +1 b-jet final state and selected benchmark beyond the standard model signals. https://zenodo.org/record/ 5126747, (2021)

3. M. Crispim Romão, N.F. Castro, R. Pedro, Finding new physics without learning about it: anomaly detection as a tool for searches at colliders (2021). arxiv:2006.05432 